SCIENTIFIC LETTER

\title{
Thrombolytic treatment in diabetic patients with acute myocardial infarction: lower rates of use than in non- diabetic patients are explained by differences in presenting ECGs
}

\author{
E Kvan, A Reikvam, INPHARM Study Investigators
}

Heart 2004;90:1346-1347. doi: 10.1136/hrt.2003.026724

$\mathrm{T}$ hrombolytic treatment lowers the mortality of patients with acute myocardial infarction (MI), and the benefit is as pronounced in diabetic patients as in non-diabetic patients. Despite this knowledge, pharmaco-epidemiological studies have reported that diabetic patients receive thrombolytic treatment less often than non-diabetic patients. The reason for this is uncertain. It has been claimed that diabetic individuals suffering acute MI do not receive optimal treatment. ${ }^{1}$ An alternative explanation is long delays from symptom onset to admission among diabetic patients, thus rendering them less suitable for thrombolysis. ${ }^{2}$ We decided to investigate more accurately the eligibility for thrombolytic treatment among diabetic patients.

\section{PATIENTS AND METHODS}

We drew a sample of patients from 16 hospitals in Norway who were discharged with a diagnosis of acute MI (ICD 10 I2 1 and I22). Patients included in the study had been hospitalised during three consecutive months between August 1999 and January 2000. Information on patient characteristics, use of thrombolytics and other cardiovascular drugs during the hospital stay, and accurate information of the ECGs on admission was collected from the hospital records 3-4 months after admission. Only patients confirmed with diabetes at admission to hospital were counted as diabetic patients. More details about the methods are given elsewhere. ${ }^{3}$

\section{RESULTS}

In the present sample of 901 patients, 121 (13\%) had diabetes. The diabetic patients were older, mean age $74.6 v$ 70.8 years $(p=0.002)$, but sex distribution was equal among diabetic and non-diabetic patients, $62.0 \% \vee 64.1 \%$ men $(p=0.66)$. Thrombolysis was used less often in the diabetic group (table 1). Significantly fewer diabetic patients presented with ST segment elevation on the ECG than nondiabetic patients, while ST segment depression was found more frequently in the diabetic population (table 1). There was a trend, although not significant, towards longer delay from symptom onset to admission among the diabetic subjects (table 1).

Among the non-diabetic patients with ST segment elevation myocardial infarction (STEMI), 61.4\% received thrombolytic treatment as compared to $51.4 \%$ of diabetic patients $(\mathrm{p}=0.24)$. Furthermore, in STEMI patients presenting within six hours of symptom onset, the proportion of patients receiving thrombolytic treatment was $77.1 \%$ in non-diabetic and $68.2 \%$ in diabetic patients, respectively $(\mathrm{p}=0.36)$.

A history of previous MI, angina pectoris, and stroke was found more frequently in the diabetic patient group $(p<0.05)$. We used logistic regression to identify independent predictors for STEMI. Age, previous MI, angina, stroke, heart failure, peripheral atherosclerosis, diabetes, and delayed admission to hospital (more than six hours) all had bivariate predictive values, positive or negative, for STEMI with $\mathrm{p}<0.10$, and were entered into a multivariate logistic regression. In the final model delayed admission (odds ratio (OR) 0.51 , 95\% confidence interval (CI) 0.37 to 0.70 ), previous MI (OR $0.48,95 \%$ CI 0.33 to 0.69 ), angina (OR $0.58,95 \%$ CI 0.41 to 0.82 ), and stroke (OR $0.48,95 \%$ CI 0.28 to 0.82 ) were independent negative predictors for STEMI. All $\mathrm{p}$ values were $<0.01$.

In a second multivariate logistic regression using a similar procedure, we identified STEMI (OR 47.58, 95\% CI 26.11 to 86.68 ) and delayed admission (OR $0.15,95 \%$ CI 0.09 to 0.24 )

\begin{tabular}{|c|c|c|c|}
\hline Patient characteristics & $\begin{array}{l}\text { *Diabetic } \\
\text { patients } \\
n=106-121\end{array}$ & $\begin{array}{l}\text { *Non-diabetic } \\
\text { patients } \\
\mathrm{n}=692-779\end{array}$ & p Value \\
\hline \multicolumn{4}{|l|}{ ECG changes at admission } \\
\hline ST elevation & $39(36.8)$ & $336(48.6)$ & 0.024 \\
\hline ST depression or T inversion & $48(45.3)$ & $240(34.7)$ & 0.034 \\
\hline Bundle branch block & $14(13.2)$ & $57(8.2)$ & 0.231 \\
\hline Normal & $5(4.7)$ & $59(8.5)$ & 0.098 \\
\hline \multicolumn{4}{|c|}{ Time from onset of symptoms to admission to hospital } \\
\hline $0-6$ hours & $55(53.9)$ & $429(60.9)$ & 0.181 \\
\hline 6 hours or more & $47(46.1)$ & $276(39.1)$ & 0.181 \\
\hline Thrombolytic treatment in hospital & $21(17.6)$ & $228(29.5)$ & 0.007 \\
\hline Alive at discharge & $95(78.5)$ & $671(86.1)$ & 0.028 \\
\hline
\end{tabular}


as the only independent predictors for thrombolytic treatment $(\mathrm{p}<0.001)$.

\section{DISCUSSION}

The less frequent use of thrombolytic treatment among diabetic patients with acute MI, as compared to non-diabetic patients, is justified. The main reason appears to be that diabetic individuals suffering acute MI present with ST segment elevation in the ECG less frequently. However, when presenting with a STEMI, diabetic patients were as likely to receive thrombolytic treatment as non-diabetic patients.

The present study did not provide proof that longer delays from onset of symptoms to admission to hospital contributed to the lower rates of thrombolysis use in diabetic subjects. Still, some contribution, as suggested by others, ${ }^{2}$ should not be ruled out, since there was a trend, although not significant, towards longer delays in diabetic patients.

In our analysis previous MI, angina pectoris, and stroke were independent negative predictors for the occurrence of STEMI. Diabetes is a generally recognised risk factor for atherosclerotic vascular disease. The influence of diabetes, which did not per se come out as an independent negative risk factor for STEMI in the multivariate analysis, is assumed to be expressed through previous MI, angina pectoris, and stroke.

The assessment and conclusion above follow from the fact that only patients with STEMI are candidates for thrombolytic treatment while patients with non-STEMI are not; furthermore, STEMI patients with less than a six hour delay are obvious candidates while longer delays weaken the indication. Dissimilarities in the atherothrombotic process in the coronary arteries between diabetic and non-diabetic subjects are assumed to be the pathophysiological basis for the clinical findings.

In the few years that have elapsed since our data were collected, percutaneous coronary intervention (PCI) has strengthened its position as a valuable tool in the management of acute MI. Today many physicians regard it as the primary method, if available in a hospital within an acceptable distance. Our findings imply that the proportion of diabetic individuals with acute MI, who are expected to be treated with PCI immediately after start of symptoms, will be lower than that of non-diabetic patients. This is because the indications for immediate PCI treatment are the same as for thrombolytic treatment.

The observed rate of thrombolytic use was relatively low. However, it did not vary greatly from the figures found in comparable studies of unselected acute MI populations. ${ }^{4}$ The proportion of STEMIs of the total number of acute MIs declined in Norway during the 1990s.

Our findings should not spur a fatalistic, conservative approach to acute MI management in diabetic individuals. Instead, the knowledge that acute MIs with ST segment elevation occur relatively infrequently suggests that the awareness is directed towards proper implementation of the guidelines for management of non-STEMI. ${ }^{5}$

\section{ACKNOWLEDGEMENTS}

We thank all the doctors who participated in the INPHARM study. ${ }^{3}$

\section{Authors' affiliations}

E Kvan, A Reikvam, I N P H A R M Study Investigators, Department of Pharmacotherapeutics, Faculty of Medicine, University of Oslo

Correspondence to: Dr Elena Kvan, Department of Pharmacotherapeutics, University of Oslo, PO Box 1065 Blindern, N-0316 Oslo, Norway; elena.kvan@labmed.vio.no

Accepted 7 February 2004

\section{REFERENCES}

1 Norhammar A, Malmberg K, Ryden L, et al. Under utilisation of evidence-based treatment partial explanation for the unfavourable prognosis in diabetic patients with acute myocardial infarction. Eur Heart $J$ 2003;24:838-44.

2 Mak KH, Topol EJ. Emerging concepts in the management of acute myocardial infarction in patients with diabetes mellitus. J Am Coll Cardiol 2000;35:563-8.

3 Reikvam A, Kvan E, Aursnes I. Use of cardiovascular drugs after acute myocardial infarction: a marked shift towards evidence-based drug therapy. Cardiovasc Drugs Ther 2002; 16:451-6.

4 European Secondary Prevention Study Group. Translation of clinical trials into practice: a European population-based study of the use of thrombolysis for acute myocardial infarction. Lancet 1996;347:1203-7.

5 Bertrand ME, Simoons ML, Fox KA, et al. Management of acute coronary syndromes in patients presenting without persistent ST-segment elevation. Eur Heart J 2002;23:1809-40. 